University of Nebraska - Lincoln

DigitalCommons@University of Nebraska - Lincoln

\title{
Prediction of a Switchable Two-Dimensional Electron Gas at Ferroelectric Oxide Interfaces
}

\author{
Manish K. Niranjan \\ University of Nebraska-Lincoln, manish@iith.ac.in \\ Yong Wang \\ University of Nebraska - Lincoln \\ Sitaram S. Jaswal \\ University of Nebraska - Lincoln, sjaswal1@unl.edu \\ Evgeny Y. Tsymbal \\ University of Nebraska-Lincoln, tsymbal@unl.edu
}

Follow this and additional works at: https://digitalcommons.unl.edu/physicstsymbal

Part of the Condensed Matter Physics Commons

Niranjan, Manish K.; Wang, Yong; Jaswal, Sitaram S.; and Tsymbal, Evgeny Y., "Prediction of a Switchable Two-Dimensional Electron Gas at Ferroelectric Oxide Interfaces" (2009). Evgeny Tsymbal Publications. 32.

https://digitalcommons.unl.edu/physicstsymbal/32

This Article is brought to you for free and open access by the Research Papers in Physics and Astronomy at DigitalCommons@University of Nebraska - Lincoln. It has been accepted for inclusion in Evgeny Tsymbal Publications by an authorized administrator of DigitalCommons@University of Nebraska - Lincoln. 


\title{
Prediction of a Switchable Two-Dimensional Electron Gas at Ferroelectric Oxide Interfaces
}

\author{
Manish K. Niranjan, Yong Wang, Sitaram S. Jaswal, and Evgeny Y. Tsymbal \\ Department of Physics and Astronomy, Nebraska Center for Materials and Nanoscience, University of Nebraska, \\ Lincoln, Nebraska 68588, USA
}

(Received 13 January 2009; published 30 June 2009)

\begin{abstract}
The demonstration of a quasi-two-dimensional electron gas (2DEG) in $\mathrm{LaAlO}_{3} / \mathrm{SrTiO}_{3}$ heterostructures has stimulated intense research activity in recent years. The 2DEG has unique properties that are promising for applications in all-oxide electronic devices. For such applications it is desirable to have the ability to control 2DEG properties by external stimulus. Here, based on first-principles calculations we predict that all-oxide heterostructures incorporating ferroelectric constituents, such as $\mathrm{KNbO}_{3} / \mathrm{ATiO}_{3}$ $(A=\mathrm{Sr}, \mathrm{Ba}, \mathrm{Pb})$, allow creating a $2 \mathrm{DEG}$ switchable between two conduction states by ferroelectric polarization reversal. The effect occurs due to the screening charge at the interface that counteracts the depolarizing electric field and depends on polarization orientation. The proposed concept of ferroelectrically controlled interface conductivity offers the possibility to design novel electronic devices.
\end{abstract}

PACS numbers: 73.20. $-\mathrm{r}, 71.30 .+\mathrm{h}, 77.80 . \mathrm{Fm}, 77.84 . \mathrm{Dy}$

Advances in thin-film deposition and characterization techniques have made possible the experimental realization of oxide heterostructures with atomically abrupt interfaces. The development of such heterostructures is very promising as it offers novel functionalities and device concepts. In particular, the recent discovery of a quasitwo-dimensional electron gas (2DEG) at the interface between two oxide insulators, $\mathrm{SrTiO}_{3}$ and $\mathrm{LaAlO}_{3}$, has attracted significant attention [1-5]. It was demonstrated that the 2DEG has high carrier mobility and electron density, which makes it interesting for applications in all-oxide field-effect devices similar to that based on modulation doping of III-V semiconductors [6]. It was also found that at low temperatures the 2DEG could become magnetic [7] or superconducting [8].

Electronic reconstruction is considered to be the primary mechanism responsible for the formation of 2DEG. Perovskite oxides have chemical formula $A^{X+} B^{Y+} \mathrm{O}_{3}$ (where $X+Y=6$ ) and along the [001] direction consist of alternating planes of $\mathrm{A}^{X+} \mathrm{O}^{2-}$ and $B^{Y+} \mathrm{O}_{2}{ }^{2-}$, which can be charged or neutral. For example, $\mathrm{LaAlO}_{3}$ consists of alternating $(\mathrm{LaO})^{+}$and $\left(\mathrm{AlO}_{2}\right)^{-}$charged planes and $\mathrm{SrTiO}_{3}$ consists of alternating $(\mathrm{SrO})^{0}$ and $\left(\mathrm{TiO}_{2}\right)^{0}$ neutral planes. When $\mathrm{LaAlO}_{3}$ is deposited on top of $\mathrm{SrTiO}_{3}$, the polar discontinuity at the interface leads to the divergence of the electrostatic potential with the increasing number of $\mathrm{LaAlO}_{3}$ unit cells. To avoid this divergence half an electron per two-dimensional unit cell is transferred across the interface from $\mathrm{LaAlO}_{3}$ to $\mathrm{SrTiO}_{3}$, causing the $\mathrm{LaO} / \mathrm{TiO}_{2}$ interface in the $\mathrm{LaAlO}_{3} / \mathrm{SrTiO}_{3}(001)$ heterostructure to be conducting [2]. Oxygen vacancies created during the growth of oxide heterostructures play an important role in contributing to high sheet conductivities [3-5]. There is, however, experimental evidence that under sufficiently high oxygen pressure electronic reconstruction becomes the dominant effect [2].
Analogous to the ionic polar discontinuity, the 2DEG may be created at an interface due to electric polarization discontinuity. This has been observed in $\mathrm{ZnO} / \mathrm{Mg}_{x} \mathrm{Zn}_{1-x} \mathrm{O}$ heterostructures where a sheet charge is formed at the interface to relax the electrostatically unfavorable state [9]. An interesting possibility is to combine the two discontinuities at the interface by using a ferroelectric oxide material as one (or both) constituents of the 2DEG heterostructure. Because of reversible polarization of the ferroelectric, such a functional interface may be served to control the 2DEG properties by ferroelectric polarization switching, which is very interesting for applications in nanoelectronics.

In this Letter, based on first-principles band structure calculations, we demonstrate the possibility to create a switchable 2DEG at oxide interfaces composed of perovskite ferroelectrics. As a representative model, we consider $\mathrm{KNbO}_{3} / \mathrm{SrTiO}_{3}$ (001) heterostructures with abrupt $\mathrm{NbO}_{2} / \mathrm{SrO}$ interfaces. $\mathrm{KNbO}_{3}$ is a ferroelectric material that consists of alternating planes of $\left(\mathrm{NbO}_{2}\right)^{+}$and $(\mathrm{KO})^{-}$ along the [001] direction, which are analogous to the polar planes in the $\mathrm{LaAlO}_{3}$. Therefore, the polar discontinuity at the $\left(\mathrm{NbO}_{2}\right)^{+} /(\mathrm{SrO})^{0}$ interface in $\mathrm{KNbO}_{3} / \mathrm{SrTiO}_{3}$ (Fig. 1) is similar to that at $(\mathrm{LaO})^{+} /\left(\mathrm{TiO}_{2}\right)^{0}$ interface in $\mathrm{LaAlO}_{3} / \mathrm{SrTiO}_{3}$. We predict, therefore, the formation of $2 \mathrm{DEG}$ at the $\mathrm{NbO}_{2} / \mathrm{SrO}$ interface. We show that electronic

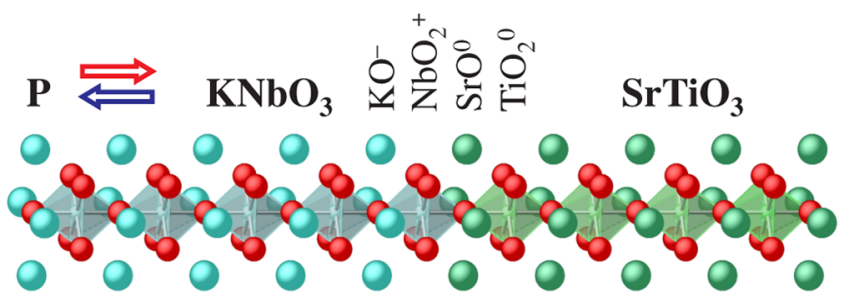

FIG. 1 (color online). Atomic structure of the $\mathrm{NbO}_{2} / \mathrm{SrO}$ interface in $\mathrm{KNbO}_{3} / \mathrm{BaTiO}_{3}(001)$ heterostructure. 
properties of the 2DEG are affected by the spontaneous polarization of ferroelectric $\mathrm{KNbO}_{3}$ due to the formation of the screening charge at the interface, counteracting the polarization charge and the depolarizing electric field. The screening charge depends on polarization orientation and consequently 2DEG can be switched between two conduction states by polarization reversal. We predict that a similar phenomenon occurs at full ferroelectric $\mathrm{KNbO}_{3} / \mathrm{BaTiO}_{3}$ and $\mathrm{KNbO}_{3} / \mathrm{PbTiO}_{3}$ oxide interfaces and infer a possibility of controllable metal-insulator transition at the interface.

First, we study the effect of ferroelectric polarization reversal on electronic properties of the $\mathrm{NbO}_{2} / \mathrm{SrO}$ interface in the $\mathrm{KNbO}_{3} / \mathrm{SrTiO}_{3}(001)$ heterostructures. Figure 1 shows the interface structure of the system. We perform density-functional calculations [10] using the projector augmented wave [11] method implemented within the Vienna $a b$ initio simulation package (VASP) $[12,13]$. We use periodic boundary conditions and we build up a $\left(\mathrm{KNbO}_{3}\right)_{m} /\left(\mathrm{SrTiO}_{3}\right)_{n}$ superlattice that contains $m=8.5$ unit cells of $\mathrm{KNbO}_{3}$ and $n=7.5$ unit cells of $\mathrm{SrTiO}_{3}$ along the [001] direction [14]. This geometry allows us to study the effect of polarization reversal in a single heterostructure by comparing properties of the two interfaces. The inplane lattice constant of the superlattice is fixed to the experimental lattice constant of $\mathrm{SrTiO}_{3}(a=3.905 \AA)$. The out-of-plane lattice constant of bulk $\mathrm{SrTiO}_{3}$ and $\mathrm{KNbO}_{3}$ are obtained by minimizing the total energy. Subsequently, the interface separation distance is determined by minimizing the total energy of the $\left(\mathrm{KNbO}_{3}\right)_{m} /\left(\mathrm{SrTiO}_{3}\right)_{n}$ superlattice keeping the in-plane lattice constant and out-of-plane separation in $\mathrm{KNbO}_{3}$ and $\mathrm{SrTiO}_{3}$ subunits fixed.

In order to reveal the presence of $2 \mathrm{DEG}$ we, first, consider the $\mathrm{KNbO}_{3} / \mathrm{SrTiO}_{3}$ interface assuming that $\mathrm{KNbO}_{3}$ is in paraelectric state. For this purpose we impose mirror plane symmetry at the central $\mathrm{NbO}_{2}$ monolayer and minimize the total energy of the whole system [15]. Figure 2(a) shows the density of states (DOS) projected onto the $4 d$ orbitals of $\mathrm{Nb}$ atoms, located at different monolayers $l$ away from the interface. It is seen that there are occupied states at and below the Fermi energy $\left(E_{F}\right)$. The electron density decreases with the distance from the interface which suggests the formation of the $n$-type $2 \mathrm{DEG}$ at the interface. A similar behavior is obtained for the Ti-3d states (Fig. 2(b)), though with a lower DOS compared to the $\mathrm{Nb}-4 d$ states. We find that the total charge transferred to the interface is $0.5 e$ per unit cell. This behavior is similar to that found previously for the $\mathrm{LaO} / \mathrm{TiO}_{2}$ interface in $\mathrm{LaAlO}_{3} / \mathrm{SrTiO}_{3}$ heterostructures [16-19].

Next we relax the constraint of reflection symmetry and minimize the total energy with respect to atomic coordinates of all the atoms in the heterostructure. This produces a ferroelectric state of $\mathrm{KNbO}_{3}$. We find that ferroelectric displacements in the central monolayers of $\mathrm{KNbO}_{3}$ are close to those found in the bulk $\mathrm{KNbO}_{3}$ that are constrained

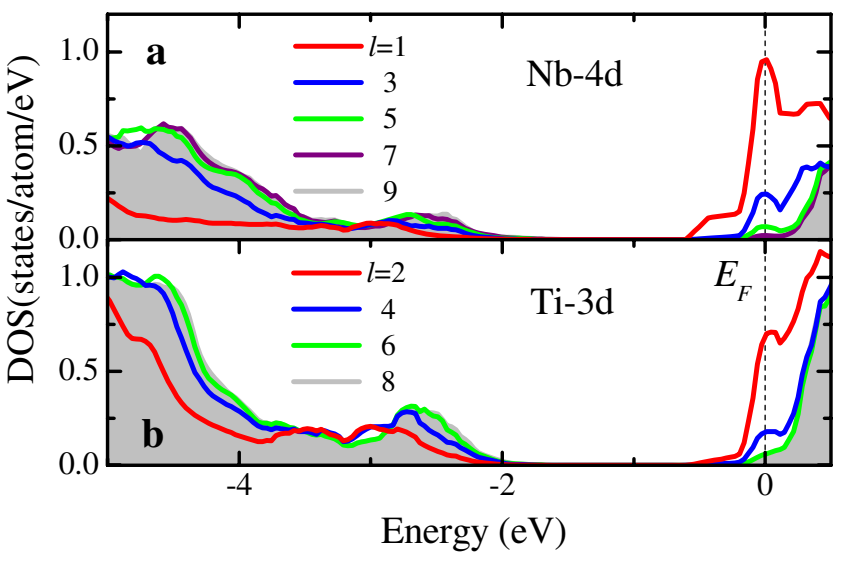

FIG. 2 (color online). Density of states (DOS) projected onto $\mathrm{Nb}-4 d$ states (a) and Ti-3d states (b) located at different monolayers $l$ away from the $\mathrm{NbO}_{2} / \mathrm{SrO}$ interface for paraelectric state of $\left(\mathrm{KNbO}_{3}\right)_{8.5} /\left(\mathrm{SrTiO}_{3}\right)_{7.5}$ superlattice. The shaded plots are the DOS of atoms in the central monolayer of $\mathrm{KNbO}_{3}$ (a) and $\mathrm{SrTiO}_{3}$ (b). The vertical line denotes the Fermi energy $\left(E_{F}\right)$.

to have the in-plane lattice constant $a=3.905 \AA$. We calculate the polarization using the Berry phase method [20] and find $P_{\mathrm{KNbO}_{3}}=0.41 \mathrm{C} / \mathrm{m}^{2}$. We also find polar displacements in $\mathrm{SrTiO}_{3}$ and estimate an induced polarization in the $\mathrm{SrTiO}_{3}$ layer to be $P_{\mathrm{SrTiO}_{3}}=0.17 \mathrm{C} / \mathrm{m}^{2}$. The energy difference between the paraelectric and ferroelectric states of $\mathrm{KNbO}_{3}$ is about $66 \mathrm{meV}$ per unit cell in the superlattice compared to $73 \mathrm{meV}$ in the bulk. This indicates that the stability of the ferroelectric state in the heterostructure is similar to that of the bulk.

In the ferroelectric state the two interfaces are no longer equivalent and are distinguished by the orientation of the ferroelectric polarization with respect to them. Here and below we assume that polarization of $\mathrm{KNbO}_{3}$ is oriented from left to right and designate these interfaces as "left" for the $\mathrm{KNbO}_{3}$ polarization pointing away from the interface and "right" for the $\mathrm{KNbO}_{3}$ polarization pointing toward the interface, as is indicated in the top panel of Fig. 3. Because of the presence of polarization the electronic properties of the two interfaces are different. This is evident from Fig. 3(a) which shows the averaged DOS at the left and right interfaces in the vicinity of the Fermi energy. It is seen that there is a significant contrast in the DOS and the number of occupied states at the two interfaces distinguished by polarization orientation. Table I shows the number of the occupied Nb-4d and Ti- $3 d$ states, which is calculated by summing up the respective local DOS on all the atoms from the middle monolayer up to the left (right) interface integrated from the conduction band minimum up to the Fermi energy. It is seen that the occupancy of both the Nb- $4 d$ states and Ti- $3 d$ states is enhanced significantly at the right interface as compared to the left interface due the presence of ferroelectric polarization in $\mathrm{KNbO}_{3}$. This suggests that carrier density of 2DEG at the interface may be controlled by switching the polarization. 

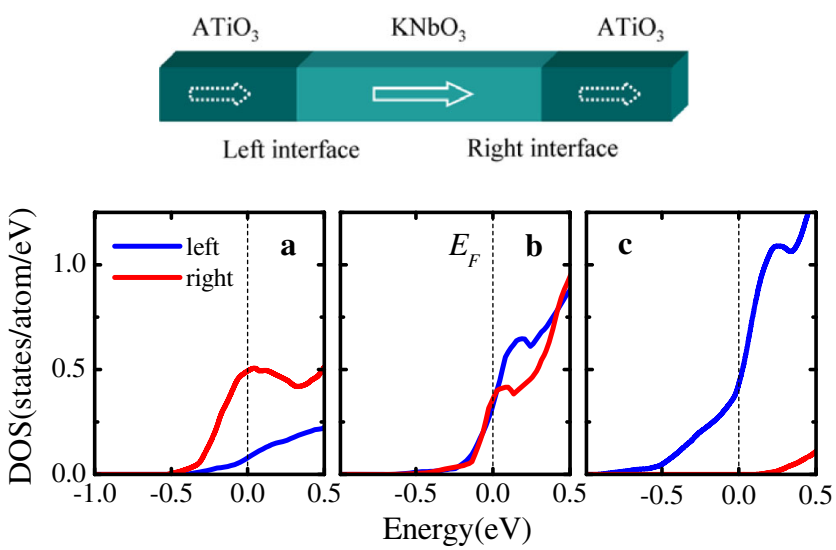

FIG. 3 (color online). DOS near the Fermi energy $\left(E_{F}\right)$ averaged over four $\mathrm{Nb}$ and three $\mathrm{Ti}$ atoms at the left and right $\mathrm{NbO}_{2} / \mathrm{AO}$ interfaces in $\left(\mathrm{KNbO}_{3}\right)_{8.5} /\left(\mathrm{ATiO}_{3}\right)_{7.5}$ superlattices for $A=\mathrm{Sr}$ (a), $A=\mathrm{Ba}$ (b), and $A=\mathrm{Pb}$ (c). The top panel shows schematically geometry of the unit cell with polarization indicated by arrows.

The physical mechanism responsible for this behavior is screening of the polarization charge which occurs through the change in the free electron density of 2DEG at the interface. The presence of screening can be seen from the comparison of the number of free carriers accumulated at the two interfaces in paraelectric and ferroelectric states of $\mathrm{KNbO}_{3} / \mathrm{SrTiO}_{3}$. We calculate local charges using WignerSeitz (WS) spheres, as specified by VASP. We find that in the paraelectric state the number of free electrons is 0.29 per interface area of the unit cell at both interfaces, whereas in the ferroelectric state the corresponding values are 0.15 and 0.43 at the left and right interfaces, respectively (see Table I). This implies that due to ferroelectricity of $\mathrm{KNbO}_{3}$ extra 0.14 electrons are accumulated at the right interface and 0.14 electrons are removed from the left interface. Evidently the induced screening change has an opposite sign to the polarization charge, thereby counteracting the depolarizing electric field. Figure 4 (circles) shows the screening charge distribution across the unit cell of $\mathrm{KNbO}_{3} / \mathrm{SrTiO}_{3}$, indicating its localization near the interfaces.

The change in the density of the $2 \mathrm{DEG}$ in going from paraelectric to ferroelectric $\mathrm{KNbO}_{3}$ can be understood from simple electrostatic considerations. As shown above, for the paraelectric state the 2DEG density at the two interfaces is the same due to the identical polar discontinuity. The ferroelectric state introduces polarization

TABLE I. Number of electrons projected on $\mathrm{Nb}$ and $\mathrm{Ti}$ atoms per interface area of the unit cell.

\begin{tabular}{lcccccc}
\hline \hline \multirow{2}{*}{ Heterostructure } & \multicolumn{3}{c}{ Right interface } & \multicolumn{3}{c}{ Left interface } \\
& $\mathrm{Nb}$ & Ti & Total & $\mathrm{Nb}$ & Ti & Total \\
\hline $\mathrm{KNbO}_{3} / \mathrm{SrTiO}_{3}$ & 0.36 & 0.07 & 0.43 & 0.09 & 0.06 & 0.15 \\
$\mathrm{KNbO}_{3} / \mathrm{BaTiO}_{3}$ & 0.25 & 0.03 & 0.28 & 0.12 & 0.15 & 0.27 \\
$\mathrm{KNbO}_{3} / \mathrm{PbTiO}_{3}$ & 0.00 & 0.00 & 0.00 & 0.25 & 0.47 & 0.72 \\
\hline \hline
\end{tabular}

charges of opposite sign at the two interfaces. This results in enhancing the polar mismatch at one interface while lowering it at the other interface which leads to a corresponding increase and decrease in the free charge density of the $2 \mathrm{DEG}$ at the two interfaces.

To make this statement quantitative we estimate the interface polarization charge density $\sigma_{p}$ from the difference of the polarizations in $\mathrm{KNbO}_{3}$ and $\mathrm{SrTiO}_{3}$. This gives the polarization charge per unit cell area of $\sigma_{p}=0.23 e$. The WS charge per unit cell at the two interfaces changes by $0.14 e$ in going from paraelectric to ferroelectric state. However, the WS charge $(0.29 e)$ underestimates the actual charge $(0.5 e)$ in the paraelectric state. When scaled with the factor $0.5 / 0.29$, the change in charge at either interface becomes $0.24 e$, which is about the same as the polarization charge. Thus, we conclude that polarization charge screening is the mechanism responsible for the change in the 2DEG carrier density.

The predicted effect of ferroelectric polarization on electronic properties of $2 \mathrm{DEG}$ at oxide interfaces is further elaborated by performing calculations for all-ferroelectric $\left(\mathrm{KNbO}_{3}\right)_{8.5} /\left(\mathrm{BaTiO}_{3}\right)_{7.5}$ and $\left(\mathrm{KNbO}_{3}\right)_{8.5} /\left(\mathrm{PbTiO}_{3}\right)_{7.5}$ heterostructures. For both systems we find that the presence of polar interfaces leads to the formation of 2DEG. However, the response of 2DEG on polarization switching is different depending on the magnitudes of ferroelectric polarization in layers comprising heterostructures.

For the $\mathrm{KNbO}_{3} / \mathrm{BaTiO}_{3}$ system, the polarization of strained $\mathrm{BaTiO}_{3}$ is similar in magnitude to the polarization of strained $\mathrm{KNbO}_{3}$. This leads to the almost cancellation of the effect of polarization on polar discontinuity at the two interfaces. This results in the reduced asymmetry of the DOS near the two interfaces, as is seen from Fig. 3(b). Figure 4 (squares) and Table I indicate that the reversal of

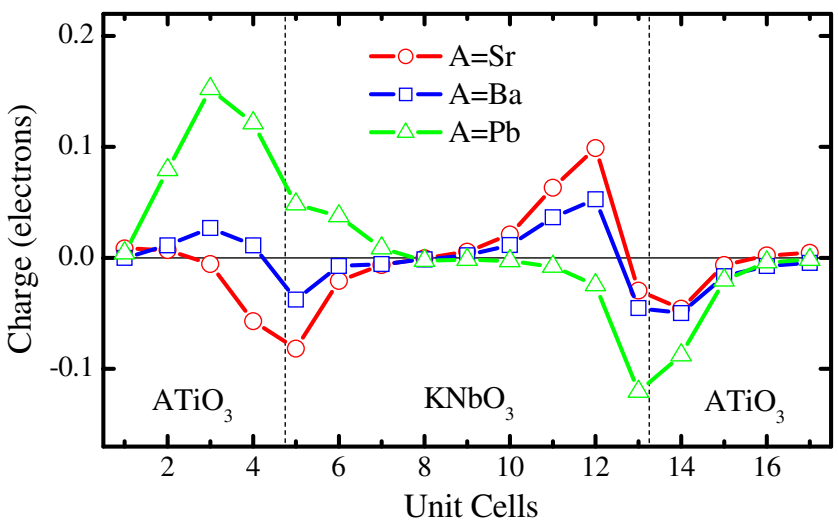

FIG. 4 (color online). Screening charge distribution across the unit cell of $\left(\mathrm{KNbO}_{3}\right)_{8.5} /\left(\mathrm{ATiO}_{3}\right)_{7.5}$ superlattices for $A=\mathrm{Sr}$ (circles), $A=\mathrm{Ba}$ (squares), and $A=\mathrm{Pb}$ (triangles). The screening charge is obtained by calculating the difference in electron density on $\mathrm{Nb}$ and $\mathrm{Ti}$ atoms for ferroelectric and paraelectric states by integrating DOS from the conduction band minimum up to Fermi energy. The direction of polarization is from left to right. 
ferroelectric polarization is accompanied by the change in the occupation of the Nb- $4 d$ and Ti- $3 d$ states, but the total number of occupied states remains almost unchanged.

For the $\mathrm{KNbO}_{3} / \mathrm{PbTiO}_{3}$ system, the polarization of the strained $\mathrm{PbTiO}_{3}$ exceeds significantly the polarization of $\mathrm{KNbO}_{3}$. This leads to a larger occupation number and the DOS at the left interface as compared to the right interface [see Fig. 3(c)], which is opposite to the case of $\mathrm{KNbO}_{3} / \mathrm{SrTiO}_{3}$ system [Fig. 3(a)]. Moreover, the polarization charge is so big (about $0.58 e$ per unit cell area) that the existing free charge at the interfaces does not fully compensate it. All the free charge is transferred from the right to the left interface making the right interface insulating, as is seen from both Fig. 3(a) and Table I. Thus, the reversal of ferroelectric polarization in $\mathrm{KNbO}_{3} / \mathrm{PbTiO}_{3}$ heterostructure leads to a metal-insulator phase transition at the interface. We note that this result is based on the generalized gradient approximation (GGA) which is known to overestimate the polarization of $\mathrm{PbTiO}_{3}$ (see, e.g., Ref. [21]). This fact does not affect, however, the very general conclusion of our calculations about the dependence of the 2DEG properties on the relative values of the electric polarization of the two constituents forming 2DEG [22].

In conclusion, based on first-principles calculations we have predicted the possibility to create a $2 \mathrm{DEG}$ in all-oxide heterostructures incorporating ferroelectric constituents, $\mathrm{KNbO}_{3} / \mathrm{ATiO}_{3}(A=\mathrm{Sr}, \mathrm{Ba}, \mathrm{Pb})$. We showed that the 2DEG is switchable between two conduction states as controlled by ferroelectric polarization orientation. This effect depends on the contrast between polarizations of the two constituents of the heterostructure: the larger is the difference in the two polarizations, the bigger is the effect. For sufficiently large polarization divergence at the interface, we predict a metal-insulator phase transition driven by polarization reversal. Experimentally, the proposed concept of ferroelectrically controlled interface conductivity could be realized, e.g., in a $\mathrm{KNbO}_{3} / \mathrm{ATiO}_{3}$ device by incorporating a gate electrode to switch the ferroelectric polarization by applied bias voltage. This concept may be very interesting for memory and logic applications, and we hope that our predictions will stimulate experimental studies in this field.

The authors thank Chang-Beom Eom and Mark Rzchowski for helpful discussions. This work was supported by the NSF (Grant No. DMR-0906443), the Nanoelectronics Research Initiative, the NSF-MRSEC (Grant No. DMR-0820521), and the Nebraska Research Initiative. Computations were performed utilizing the Research Computing Facility of the University of Nebraska-Lincoln.

[1] A. Ohtomo and H. Y. Hwang, Nature (London) 427, 423 (2004).
[2] N. Nakagawa, H. Y. Hwang, and D. A. Muller, Nature Mater. 5, 204 (2006).

[3] G. Herranz, M. Basletic, M. Bibes, C. Carretero, E. Tafra, E. Jacqet, K. Bouzehouane, C. Deranlot, A. Hamzic, J.-M. Broto, A. Berthelemy, and A. Fert, Phys. Rev. Lett. 98, 216803 (2007).

[4] W. Siemons, G. Koster, H. Yamamoto, W. A. Harrison, G. Lucovsky, T. H. Geballe, D. H. A. Blank, and M. R. Beasley, Phys. Rev. Lett. 98, 196802 (2007).

[5] A. Kalabukhov, R. Gunnarson, J. Borjesson, E. Olsson, T. Claeson, and D. Winkler, Phys. Rev. B 75, 121404(R) (2007).

[6] S. Thiel, G. Hammerl, A. Schmehl, C. W. Schneider, and J. Mannhart, Science 313, 1942 (2006).

[7] A. Brinkman, M. Huijben, M. Van Zalk, J. Huijban, U. Zeitler, J. C. Maan, W. G. Van der Wiel, G. Rijnders, D. H. A. Blank, and H. Hilgenkamp, Nature Mater. 6, 493 (2007).

[8] N. Reyren, S. Thiel, A. D. Caviglia, L.F. Kourkoutis, G. Hammerl, C. Richter, C. W. Schneider, T. Kopp, A.-S. Ruetschi, D. Jaccard, M. Gabay, D. A. Muller, J.-M. Triscone, and J. Mannhart, Science 317, 1196 (2007).

[9] T. Kamiya and M. Kawasaki, MRS Bull. 33, 1061 (2008).

[10] W. Kohn and L. J. Sham, Phys. Rev. 140, A1133 (1965).

[11] P. E. Blöchl, Phys. Rev. B 50, 17953 (1994).

[12] G. Kresse and J. Furtmuller, Phys. Rev. B 54, 11169 (1996).

[13] The exchange-correlation functional is approximated using the Perdew-Burke-Ernzerhoff form of the GGA along with a standard plane wave basis set with a kinetic energy cutoff of $500 \mathrm{eV}$. The bulk and superlattice calculations are performed using the $6 \times 6 \times 6$ and $6 \times 6 \times 1$ Monkhorst-Pack $k$-point mesh, respectively. The calculations are converged to $10^{-4} \mathrm{eV} /$ cell and the structures are relaxed until the largest force becomes less than $0.02 \mathrm{eV} / \AA$.

[14] We have also performed calculations for supercells composed of $m=6.5,8.5$, and 10.5 unit cells of $\mathrm{KNbO}_{3}$ and $n=m-1$ unit cells of $\mathrm{SrTiO}_{3}$ and found that the results are qualitatively similar.

[15] C.-G. Duan, R. Sabirianov, W.-N. Mei, S. S. Jaswal, and E. Y. Tsymbal, Nano Lett. 6, 483 (2006).

[16] Z. S. Popovic and S. Satpathy, Phys. Rev. Lett. 94, 176805 (2005); Z. S. Popović, S. Satpathy, and R. M. Martin, Phys. Rev. Lett. 101, 256801 (2008).

[17] M.S. Park, S. H. Rhim, and A. J. Freeman, Phys. Rev. B 74, 205416 (2006).

[18] R. Pentcheva and W. E. Pickett, Phys. Rev. B 74, 035112 (2006); Phys. Rev. Lett. 99, 016802 (2007).

[19] K. Janicka, J.P. Velev, and E. Y. Tsymbal, J. Appl. Phys. 103, 07 B508 (2008); Phys. Rev. Lett. 102, 106803 (2009).

[20] R. D. King-Smith and D. Vanderbilt, Phys. Rev. B 47, 1651 (1993).

[21] Y. Umeno, B. Meyer, C. Elsässer, and P. Gumbsch, Phys. Rev. B 74, 060101(R) (2006).

[22] Our calculations within the local density approximation for the $\mathrm{KNbO}_{3} / \mathrm{SrTiO}_{3}$ interface show that the predicted results are qualitatively similar to those of the GGA. 